\title{
En un accidente de tráfico ¿las lesiones pueden ayudar a dictaminar si llevaba el cinturón puesto?.
}

In a traffic accident, injuries may help to establish if the safety belt was used?.

\section{A. Ortega Pérez ${ }^{1}$}

\section{RESUMEN}

En dos juicios distintos se discutió sobre si las accidentadas llevaban puesto el cinturón de seguridad en el momento del accidente. Las características del accidente y las de las lesiones, junto a la revisión de la bibliografía médica nacional e internacional sobre el asunto llevan a concluir que las fracturas esternales y costales tienen una gran posibilidad de haber sido causadas por el cinturón, especialmente cuando el accidentado no presenta lesiones en el cráneo, en la cara, en el abdomen o en las extremidades superiores. Asimismo, también aumentan con la edad los riesgos de fracturas esternales, costales o ambas.

Palabras clave: Accidente de tráfico, cinturón de seguridad, fractura esternal, fractura costal, lesiones, traumatología.

Cuad Med Forense 2005; 11(39):35-42

\section{ABSTRACT}

In two different Courts, the lawyers asked if the injured person was using the safety belt when the accident happened. The characteristics of the accident and those of the injuries, along with the review of the national and international medical literature about the question lead to the conclusion that sternal and rib fractures have a great possibility to have been caused by the safety belt itself, specially when the person does not have injuries in the cranium, in the face, in the abdomen or in the arms. On the same way, the risk of sternal and/or costal fractures increases with the age of the person.

Key words: Lesion, safety belt, rib fracture, sternal fracture, traffic accident, traumatology.

El autor no ha recibido ninguna subvención para elaborar este trabajo.

Correspondencia: Dr. Arturo Ortega. Área de Medicina Legal y Forense. Facultad de Medicina y Ciencias de la Salud. C/ Sant Llorenç, 21. 43201 - Reus (Tarragona). Correo electrónico: arturo.ortega@urv.net

1 Doctor en Medicina. Profesor de Medicina Legal y Toxicología. Facultad de Medicina y Ciencias de la Salud. Universidad "Rovira i Virgili". 


\section{INTRODUCCIÓN:}

La importancia sanitaria de los accidentes de tráfico en España es innegable, si se arguyen los tres mil quinientos a cuatro mil muertos y los ciento treinta mil heridos que producen dichos accidentes cada año. Las investigaciones sobre estos accidentes han permitido comprobar afirmaciones tan repetidas como el agravamiento de las lesiones con el aumento de la violencia del accidente o con la edad cronológica del accidentado [1,2].

No es este el foro para discutir la importancia relativa de cada una de sus causas: las carreteras insuficientes, los problemas súbitos e inevitables ocurridos durante la conducción o, principalmente, los hábitos imprudentes de los conductores, de los ciclistas y de los peatones. Sin embargo, es cierto que a pesar de las campañas y de la obligación legal de llevar puesto el cinturón de seguridad cuando se viaja en un automóvil, no lo llevan entre un tercio y la mitad de los ocupantes de vehículos implicados en un accidente de tráfico con lesionados. Según una encuesta reciente a los conductores españoles, nuestros jóvenes usan o no el cinturón en función de sus percepciones sobre la seguridad que les dará y sobre las molestias que les causará su uso y también por la influencia del grupo social; en cambio, la acción represiva de las multas por no llevarlo apenas les preocupa [3].

Y todo ello a pesar de las ventajas probadas del uso del cinturón: muchos estudios han demostrado que reduce las lesiones de los viajeros -los conductores y sus acompañantes-, en comparación con las sufridas por quienes no lo usaron. Sin embargo, no puede pretenderse que utilizando el cinturón se pueda salir indemne de un accidente grave, porque la energía cinética de la persona en movimiento -su masa por el cuadrado de su velocidad- ha de ser contrarrestada en décimas de segundo. Para hacernos una idea, el vehículo que circula a $60 \mathrm{~km} / \mathrm{hora}$ y choca contra un obstáculo inmóvil sufre el mismo choque que cayendo desde 28 metros de altura. Aunque la deformación del vehículo absorbe buena parte de esa energía, el resto se ejercerá sobre el cuerpo del accidentado y la estructura que se opone a la energía cinética de este es una banda relativamente estrecha, aplicada contra el esternón y contra las costillas.

Para no alargar demasiado esta introducción, los hechos básicos sobre las ventajas del uso de los cinturones se recogen en la Tabla I.

Tabla 1.- Hechos básicos relacionados con la evitación de lesiones por accidentes de tráfico.

- El uso del cinturón de seguridad reduce la gravedad de las lesiones de los pasajeros que viajan en los asientos delanteros [1,4,5].

- El cinturón que lleva tres puntos de anclaje y que fija el hombro y la cintura reduce el riesgo de muerte hasta siete veces respecto al de quienes no llevan cinturón [6].

- Estar en los asientos posteriores del vehículo reduce el riesgo de muerte o de lesión grave en más de un 30 \% en caso de colisión frontal, en comparación con quienes viajan en los asientos delanteros $[7,8]$.

- En los asientos posteriores, los cinturones que sujetan también el hombro son más seguros que aquellos que sólo fijan la pelvis $[9,10]$.

- Los pasajeros del asiento trasero que no van sujetos se desplazan con el accidente y aumentan el riesgo de lesiones y de muerte para los de los asientos delanteros: el riesgo de muerte de estos se multiplica por 1,75 [10] o hasta por 4,8 [11]. 
Dadas esas ventajas, no es extraño que el uso de cinturón de seguridad en el momento del accidente comience a tenerse en cuenta a la hora de repartir las responsabilidades de las lesiones sufridas en este: si el ocupante no lo usaba, será en parte responsable de los daños corporales padecidos. La inclusión de este hecho como modificador de las sentencias hace que cada día sea más común la discusión sobre si el afectado lo llevaba o no abrochado.

Para contestar a esa pregunta el juzgador cuenta con distintas pruebas, desde la declaración del lesionado hasta las testificales, el atestado o las pruebas médicas. Las dos primeras pueden estar mediatizadas, en un sentido o en el otro, por el interés comprensible en reducir la culpa propia en las lesiones ocurridas. El atestado, por su parte, tiene presunción de veracidad, pero raramente los agentes habrán estado allí y tenido la ocasión de ver por sí mismos si el lesionado llevaba puesto o no el cinturón en el momento del accidente.

Y todo esto nos lleva al papel de las pruebas médicas como ayuda para la decisión sobre el uso del cinturón de seguridad, que es el objeto de esta pequeña reflexión. A partir de dos casos en los cuales en el transcurso del juicio se discutió sobre el hecho citado, se expondrá aquí la situación médica de los lesionados y se revisará la bibliografía médica para concluir a favor o en contra, dentro de lo posible, de que los afectados llevaran el cinturón de seguridad en esos accidente. Confiamos en que los datos aportados puedan ser útiles a los médicos forenses para resolver casos similares.

\section{LOS CASOS CLÍNICOS:}

Para cada uno de ellos se presentarán los datos sumariales, policiales, ambientales y médicos de interés para poder evaluarlos, evitando a la vez aquellos datos innecesarios y que puedan llevar a la identificación personal de los lesionados.

\section{CASO N. ${ }^{\circ}$}

- Datos sumariales: La lesionada era una mujer de cincuenta años de edad que viajaba en el asiento delantero derecho de un turismo a una velocidad de unos $50 \mathrm{~km} / \mathrm{hora}$. Una furgoneta que circulaba a una velocidad similar en sentido contrario chocó contra el ángulo delantero izquierdo del turismo.

- Datos policiales: Los policías llegaron poco después y levantaron el atestado correspondiente, pero no pudieron informar sobre el uso del cinturón porque la mujer había podido salir por su propio pie del vehículo. El conductor de la furgoneta declaró que la afectada no llevaba puesto el cinturón de seguridad y ella manifestó que sí lo había usado.

- Datos ambientales: ocurrió una mañana de marzo en una carretera local con rectas cortas y abundantes curvas.

- Datos médicos: La mujer sufrió fracturas en el esternón y en la unión condrocostal de la décima y la undécima costillas izquierdas. En el informe de la atención en el Servicio de Urgencias no consta que hubiera lesiones cutáneas en el área cubierta por el cinturón, ni que padeciera lesiones en la cara, en la cabeza o en las manos.

\section{CASO N. ${ }^{\circ} 2$}

- Datos sumariales: La lesionada era una mujer de veinticinco años de edad, que viajaba en el asiento posterior izquierdo de un turismo de categoría media-alta, a una velocidad superior a los cien kilómetros por hora. Sin intervención de ningún agente externo, el conductor perdió el control de su vehículo, el cual se desvió a la derecha, chocó casi frontalmente contra un talud de roca y después giró sobre su eje vertical. 
- Datos policiales: Cuando los agentes llegaron encontraron a los pasajeros de los asientos traseros acostados en el suelo, alineados en el arcén de la autopista, según el dibujo del atestado; anotaron que estos afectados no llevaban el cinturón en el momento del accidente, posiblemente porque interpretaron que habían salido despedidos del vehículo. Sí se habían disparado los cojines de aire de ambos asientos delanteros.

- Datos ambientales: ocurrió una mañana de agosto, en una autopista.

- Datos médicos: El conductor y el copiloto resultaron ilesos; de los tres que viajaban detrás, uno murió, otro quedó tetrapléjico y la otra es la joven cuya situación relatamos en los párrafos siguientes.

La trasladaron al hospital, donde ingresó con una puntuación de 7 en la escala de coma de Glasgow y con volet costal con dificultad respiratoria, que los traumatólogos atribuyeron a fracturas condrocostales bilaterales. Le apreciaron una luxación anterior de la articulación coxofemoral derecha, pero en los informes médicos o de enfermería de Urgencias no registraron la presencia de contusiones, erosiones o heridas cutáneas, ni las que habrían estado bajo el área del cinturón ni las que podrían achacarse al choque contra los cristales o al rozamiento contra el suelo.

Por último, en la TAC de seguimiento que practicaron a la lesionada a las 24 horas del accidente le diagnosticaron un infarto de la arteria cerebral media derecha. El ecodoppler transcraneal mostró una "oclusión de la arteria carótida derecha" "Posible disección-oclusión traumática de la arteria carótida interna derecha."

\section{ESTUDIO BIBLIOGRÁFICO Y DISCUSIÓN DE LOS CASOS:}

Para poder peritar en cada uno de estos casos a partir de los datos médicos han de buscarse los resultados de tres tipos de estudios: los que describen las lesiones que pueden atribuirse exclusivamente a la sujeción por el cinturón de seguridad, los que relacionan las lesiones cutáneas y las lesiones internas bajo aquellas y, por último, a la relación entre las fracturas esternales o las lesiones del cuello con el uso del cinturón de seguridad.

\section{Las lesiones específicas causadas por el uso del cinturón de seguridad.}

En los párrafos siguientes se explican los resultados de los estudios existentes sobre las lesiones atribuibles al cinturón de seguridad y en la Tabla 2 se resumen sus conclusiones más prácticas.

Tabla 2.- Cuestiones prácticas sobre las lesiones atribuibles al cinturón de seguridad.

- El hecho de sufrir lesiones moderadas o graves a consecuencia del accidente no excluye que se llevara abrochado el cinturón en ese momento, especialmente si este fue muy violento.

- No siempre existen, o se registran, lesiones cutáneas en los portadores de cinturón, pero cuando las hay deben buscarse lesiones internas bajo ellas.

- La lesión más común en los usuarios de cinturón es la fractura esternal.

- El riesgo de lesión torácica moderada aumenta claramente con la edad.

- El uso de un cinturón con tres puntos de anclaje reduce significativamente el riesgo de lesiones en la cabeza, en la cara y en el abdomen y aumenta el de fracturas esternales y costales, aunque estas son menos graves que las de quienes no van sujetos.

Resumiendo en dos líneas, las fracturas "bajo el cinturón" -esternales y costales- apoyan que se usó este, mientras que las lesiones en la cabeza, en la cara y en el abdomen, apoyan el que no se usara. Esta regla sencilla puede aplicarse a los choques frontales o a los alcances, pero no a los laterales o a las vueltas de campana. 
Hill et al. [13] recogieron datos de 1025 personas con lesiones atribuibles exclusivamente al cinturón de seguridad y averiguaron que el 19,4\% habían sufrido lesiones toracoabdominales clasificadas en los niveles 2 o superiores en la Escala Abreviada de Lesiones (Abbreviated Injury Scale o AIS), entre las cuales la fractura esternal había sido la lesión más común. Sólo el 4,5% de los implicados sufrieron unas lesiones graves, clasificables como de niveles 3 o superiores de la AIS. Los accidentados con un riesgo mayor de lesiones graves por el cinturón de seguridad habían sido las mujeres de 70 años de edad o más, pero los efectos de la edad fueron mucho más claros en las personas con lesiones torácicas clasificadas en el nivel 2 de la AIS. El factor más claramente relacionado con esas lesiones torácicas graves era la magnitud de la deceleración a consecuencia del impacto.

Otros autores llegaron también a la conclusión de que las fracturas esternales y costales en los sujetados por el cinturón son más comunes entre los ancianos que entre los jóvenes, algo que parece lógico $[14,15]$.

Hace ya veinticinco años, un estudio sueco [16] comparó las lesiones sufridas por los conductores sujetos mediante el cinturón con aquellas padecidas por quienes no lo llevaban abrochado; los primeros habían sufrido un número significativamente menor de lesiones en la cabeza o en el cuello y un número significativamente mayor de lesiones torácicas, aunque estas eran menos graves que las de quienes no iban sujetos por el cinturón de seguridad.

Ese patrón de lesiones se ha ido repitiendo en otras investigaciones: algunas fracturas de clavícula, de esternón y de costillas y menos lesiones de las vértebras cervicales, del abdomen y de la pelvis, del cráneo y del cerebro [17], así como de la cara [ I 8]. Koushki et al. [4] hallaron que quienes no llevaban puesto el cinturón sufrieron más a menudo lesiones de la cabeza, de la cara, del abdomen y de los miembros; en cambio, quienes lo llevaban puesto sufrieron con más frecuencia lesiones del cuello y del pecho. También Bradbury y Robertson [I] concluyeron que los cinturones de seguridad podían aumentar el riesgo de lesión del cuello, aunque reducían la gravedad de las lesiones en la cara y en el pecho.

Por el contrario, Porter y Zhao [19] apenas encontraron un patrón específico al comparar a 376 personas que estaban sujetas por el cinturón con otras 544 que no lo estaban: los primeros doblaron la incidencia de fractura esternal y redujeron en un tercio la de lesión en la cabeza. La conclusión de los autores fue que a todos los accidentados se les deben buscar con cuidado lesiones en todas las regiones corporales.

Sin embargo, las fracturas esternales o costales pueden pasar desapercibidas, dado que, especialmente las segundas, son difícilmente detectables en las radiografías [20]. Por eso es necesario prestar atención a los pacientes que continúan quejándose de dolor torácico anterior o lateral unas semanas después del accidente.

Otra investigación comparó la protección brindada por los cinturones con tres puntos de fijación con la de los que sólo sujetan las caderas [2।]. Entre los primeros, las lesiones más comunes afectaron al pecho y a la cintura escapular y entre los segundos, las que implicaban al abdomen y a las vértebras.

\section{La relación entre lesiones cutáneas y las internas entre los usuarios del cinturón de seguridad.}

La presencia de abrasiones en las zonas del cuello, del pecho y del abdomen en contacto con el cinturón es una prueba irrefutable del uso de este, pero, como decía un médico forense, no encontrarlas no prueba que el afectado no lo llevara, porque "la ausencia de pruebas no prueba la ausencia". Y aunque algunos artículos refieren que casi siempre se hallan [2I], es posible que esas abrasiones simplemente no se registraran en los informes médicos, tal vez porque en la constelación 
de lesiones presentes parecían carecer de importancia. Por el contrario, sí suelen referirse a ellas los diplomados de enfermería en sus hojas de atención, porque llevan a cabo las curas tópicas precisas.

Los médicos clínicos están más preocupados por si las lesiones cutáneas indican lesiones musculares, viscerales u óseas. Hayes et al. concluyeron que el 30 \% de quienes sufrían lesiones cutáneas también las tenían internas [22]; incluso relacionaron las abrasiones en el cuello con lesiones de la arteria carótida, de la laringe y de la columna cervical, las abrasiones del pecho con fracturas del esternón, de las costillas y de las clavículas, además de lesiones cardíacas y de la aorta torácica, y las abrasiones abdominales con desgarros mesentéricos, con perforación y hematoma intestinales, con fracturas de Chance y con lesiones de la aorta abdominal. Por eso recomendaron buscar con diligencia lesiones internas a quienes sufrieran esas abrasiones cutáneas por el cinturón.

\section{El papel de las fracturas esternales como marcador del uso de cinturón de seguridad.}

De entrada, las fracturas esternales son poco frecuentes o sea que en todo caso serían un marcador poco útil. Otremski et al. [23] las hallaron sólo en el 3,7 \% de las 2097 víctimas consecutivas de accidentes de tráfico que habían necesitado atención hospitalaria. Las fracturas esternales habían sido más frecuentes en viajeros sujetos por el cinturón, en los implicados en un choque frontal y en los de más edad.

Las observaciones clínicas sobre fracturas esternales en portadores de cinturón, que se vienen publicando desde hace más de veinte años [24,25,26], nos permiten cuando menos sospechar la relación entre ambos hechos. Una publicación recoge una situación muy similar a la presentada en el primer caso clínico, la fractura esternal ocurrida a una persona que viajaba como copiloto en un vehículo que se movía a velocidad moderada [27].

Más allá de esa primera llamada de atención, distintas publicaciones relacionan la sujeción por el cinturón con las fracturas esternales, que se han hecho más frecuentes con la introducción de la obligación legal de su uso [28]. Ya hemos citado que la fractura esternal fue la lesión predominante en personas cuyas lesiones, de nivel 2 o superior en la AIS, podían atribuirse exclusivamente a la sujeción por el cinturón de seguridad [I].

Roy-Shapira et al. revisaron los registros de 28 pacientes con fracturas esternales y 22 de ellos habían usado cinturón de seguridad [29].

Sin embargo, no todas las investigaciones aprecian esta correlación entre el uso del cinturón y las fracturas esternales: Newman [30] encontró más lesiones de tejidos blandos en los individuos sujetos, pero un número similar de fracturas claviculares o esternales. El uso de cinturón tampoco había reducido la incidencia global de costillas rotas, pero sí el de personas con lesiones graves -cuatro o más fracturas costales-.

Las fracturas costales, cuando ocurren, suelen estar situadas bajo el cinturón de seguridad [3 I], aunque la correlación no es completa, porque también influye la mecánica del accidente. Los determinantes más intensos del riesgo de fracturas costales son la fuerza de deflexión aplicada sobre el tórax -es decir, la gravedad de la colisión-, y la edad del implicado [32].

\section{Las lesiones en el cuello y el uso de cinturón de seguridad.}

Las lesiones en el cuello que podrían relacionarse con el uso de cinturón de seguridad son las disecciones carotídeas traumáticas y las lesiones laríngeas. Las primeras son lesiones muy raras, como explican Mayberry et al. [33]. Estos autores recogieron los datos de 35.212 pacientes ingresados en un hospital que padecían contusiones en el cuello y de ellos, sólo a 17 (el 0,05 \%) se les diagnosticó una lesión carotídea. 
Sin embargo, dentro de la rareza, el "signo del cinturón de seguridad en el cuello" aumenta el riesgo de disección carotídea traumática: DiPerna et al. [34] recogieron 131 casos de ese signo en personas que habían sufrido un accidente de tráfico y sólo una de ellas (0,76 \%) había sufrido una estenosis carotídea grave; fue una persona con muchas lesiones en la cara, en la cabeza y en el pecho, con signos de lesión neurológica focal y con una puntuación en la escala de Glasgow del coma de 8.

Aún con todo, se han publicado varias observaciones clínicas de disecciones traumáticas de la arteria carótida interna a consecuencia de un accidente de tráfico, en los que el lesionado estaba sujeto por el cinturón de seguridad $[35,36]$. En dos casos de oclusión traumática de la arteria carótida interna derecha [37], en los cuellos no se apreciaban abrasiones ni equimosis, ambas oclusiones se produjeron unos $3 \mathrm{~cm}$ más allá de la bifurcación y los afectados conducían los vehículos, lo que significa que la lesión se produjo al lado contrario del que tocaba al cinturón. Otro accidentado, que llevaba un cinturón que sólo sujetaba el hombro, sufrió la lesión de la carótida común y una sección transversal de la tráquea [38].

Si se tiene en cuenta la rareza de esas disecciones traumáticas hemos de concluir que el grado de relación que podemos atribuir a esa lesión con el uso de cinturón supera el nivel de la simple sospecha.

Pero no son esas las únicas lesiones del cuello que pueden afectar a usuarios del cinturón: se han publicado observaciones clínicas de hematomas bilaterales en las cuerdas vocales [39], de fractura del cartílago tiroideo [40] e incluso de estrangulaciones debidas al uso de cinturones que sujetan el hombro, pero no la cadera [4I]. Todo ello parece significativo dada la rareza de dichas lesiones.

En definitiva, después del análisis del accidente y el de las características de las lesiones, así como tras la revisión bibliográfica sobre esos puntos, la conclusión sobre los casos clínicos planteados debe ser la siguiente:

- Para el caso número uno: la fractura esternal y de la unión condrocostal izquierda de las costillas $10^{a}$ y $1 \mathrm{I}^{\mathrm{a}}$ en una mujer de 50 años que viajaba como copiloto y que no sufrió otras lesiones en la cara, en el cráneo o en las extremidades superiores se debió sin duda al cinturón de seguridad.

- La conclusión es similar para el caso número dos: las fracturas condrocostales bilaterales y la disección-oclusión traumática de la arteria carótida interna derecha en una mujer joven que viajaba en el asiento trasero izquierdo del turismo que sufrió un accidente muy violento y que no sufrió otras lesiones en la cara, en el cráneo o en las extremidades superiores ni tampoco erosiones o equimosis indica que llevaba el cinturón de seguridad puesto.

\section{BIBLIOGRAFÍA}

I. Bradbury A, Robertson C. Prospective audit of the pattern, severity and circumstances of injury sustained by vehicle occupants as a result of road traffic accidents. Arch Emerg Med 1993;10:15-23.

2. Miltner $\mathrm{E}$, Kallieris $\mathrm{D}$, Zimmer $\mathrm{G}$. The influence of age on injury severity of restrained front seat occupants in head-on collisions. Med Law 1995;14:105-16.

3. Cunill M, Gras ME, Planes M, Oliveras C, Sullman MJ. An investigation of factors reducing seat belt use amongst $S p a n i s h$ drivers and passengers on urban roads. Accid Anal Prev 2004;36:439-45.

4. Christian MS. Morbidity and mortality of car occupants: comparative survey over 24 months. Br Med J (Clin Res Ed) 1984;289:1525-6.

5. Koushki PA, Bustan MA, Kartam N. Impact of safety belt use on road accident injury and injury type in Kuwait. Accid Anal Prev 2003;35:237-41.

6. Rivara FP, Koepsell TD, Grossman DC, Mock C. Effectiveness of automatic shoulder belt systems in motor vehicle crashes. JAMA 2000;283:2826-8.

7. Huelke DF, Compton CP. The effects of seat belts on injury severity of front and rear seat occupants in the same frontal crash. Accid Anal Prev 1995;27:835-8.

8. Smith KM, Cummings P. Passenger seating position and the risk of passenger death or injury in traffic crashes. Accid Anal Prev 2004;36:257-60. 
9. Woelfel GF, Moore EE, Cogbill TH, Van Way CW 3rd. Severe thoracic and abdominal injuries associated with lap-harness seatbelts. J Trauma 1984;24:166-7.

10. Robertson LS. Reduced fatalities related to rear seat shoulder belts. Inj Prev 1999;5:62-4.

II. Broughton J. The actual threat posed by unrestrained rear seat car passengers. Accid Anal Prev 2004;36:627-9.

12. MacLennan PA, McGwin G Jr, Metzger J, Moran SG, Rue LW 3rd. Risk of injury for occupants of motor vehicle collisions from unbelted occupants. Inj Prev 2004;10:363-7.

13. Hill JR, Mackay GM, Morris AP. Chest and abdominal injuries caused by seat belt loading. Accid Anal Prev 1994;26:11-26.

14. Hendey GW, Votey SR. Injuries in restrained motor vehicle accident victims. Ann Emerg Med 1994;24:77-84.

15. Martinez R, Sharieff G, Hooper J. Three-point restraints as a risk factor for chest injury in the elderly. J Trauma 1994;37:980-4.

16. Mellbring G, Dahlin S, Lindblad B. The hospital experience of seat belt legislation in the county of Skaraborg, Sweden. Injury 1981;12:506-9.

17. Teifke A, Degreif J, Geist M, Schild H, Strunk H, Schunk K. The safety belt: effects on injury patterns of automobile passengers. Rofo 1993;159:278-83.

18. Cox D, Vincent DG, McGwin G, MacLennan PA, Holmes JD, Rue LW 3rd. Effect of restraint systems on maxillofacial injury in frontal motor vehicle collisions. J Oral Maxillofac Surg 2004;62:571-5. 19. Porter RS, Zhao N. Patterns of injury in belted and unbelted individuals presenting to a trauma center after motor vehicle crash: seat belt syndrome revisited. Ann Emerg Med 1998;32:418-24.

20. LaBan MM, Siegel CB, Schutz LK, Taylor RS. Occult radiographic fractures of the chest wall identified by nuclear scan imaging: report of seven cases. Arch Phys Med Rehabil 1994;75:353-4.

21. Girelli G, Di Natale R, Manocchi MC, Lutman M. Seat belt syndrome. Radiol Med (Torino) 1993;86:444-52.

22. Hayes CW, Conway WF, Walsh JW, Coppage L, Gervin AS. Seat belt injuries: radiologic findings and clinical correlation. Radiographics 1991;II: 23-36.

23. Otremski I, Wilde BR, Marsh JL, McLardy Smith PD, Newman RJ. Fracture of the sternum in motor vehicle accidents and its association with mediastinal injury. Injury 1990;21:81-3.

24. O'Malley MK, Duignan JP, Lavelle JS. Fractured sternum associated with the use of seat belts. Ir Med ] 1983;76:131-2.

25. Evans PD, Mackie IG. Fracture of the body of the sternum associated with the use of static seat belts. Injury 1985;16:485-6.
26. Coel M, Sato G. Sternal fractures in motor vehicle accident victims restrained by lap-shoulder safety belts: can bone scintigraphy distinguish passenger from driver? Clin Nucl Med 2000;25:140-I.

27. Restifo KM, Kelen GD. Case report: sternal fracture from a seatbelt. J Emerg Med 1994;I2:32I-3.

28. Purkiss SF, Graham TR. Sternal fractures. Br J Hosp Med 1993;50:107-12.

29. Roy-Shapira A, Levi I, Khoda J. Sternal fractures: a red flag or a red herring? J Trauma 1994;37:59-61.

30. Newman RJ. Chest wall injuries and the seat belt syndrome. Injury 1984;16: II0-3.

3I. Arajarvi E, Santavirta S. Chest injuries sustained in severe traffic accidents by seatbelt wearers. J Trauma 1989;29:37-41.

32. Kent R, Patrie J. Chest deflection tolerance to blunt anterior loading is sensitive to age but not load distribution. Forensic Sci Int 2005;149:121-8.

33. Mayberry JC, Brown CV, Mullins RJ, Velmahos GC. Blunt carotid artery injury: the futility of aggressive screening and diagnosis. Arch Surg 2004;139:609-12.

34. DiPerna CA, Rowe VL, Terramani TT, Salim A, Hood DB, Velmahos GC, Weaver FA. Clinical importance of the "seat belt sign" in blunt trauma to the neck. Am Surg 2002;68:44I-5.

35. Reddy K, Furer M, West M, Hamonic M. Carotid artery dissection secondary to seatbelt trauma: case report. J Trauma 1990;30:630-3.

36. Mason DP, Orgill DP, Schoen F], Rizzo RJ. Traumatic carotid artery dissection of restrained driver and thoracic aorta transection of unrestrained passenger in a motor vehicle accident: case report. J Trauma 1997; 43:537-40.

37. Watanabe Y, Mezaki T, Yamamoto Y, Kuzuhara S, Ueyama M. Internal carotid artery occlusion related to seat belt shoulder strap: report of two cases. Rinsho Shinkeigaku 1996;36:670-4.

38. McConnell EJ, Macbeth GA. Common carotid artery and tracheal injury from shoulder strap seat belt. J Trauma 1997;43:150-2.

39. De Lorenzo RA, Mayer D, Gardner GM. Bilateral vocal cord hematomas associated with shoulder harness use. Am J Emerg Med 1991;9:158-60.

40. Steuart RD, Morrison RT. Fracture of the laryngeal cartilage. An incidental finding on bone scintigraphy. Clin Nucl Med 1992;17:815-7.

4I. Veenema KR. Strangulation associated with a passive restraint shoulder harness seatbelt: case report. J Emerg Med 1994;12:317-20. 